\title{
Renal neoplasms in patients with renal cystic diseases
}

\author{
E. A. P. Reichard, M. A. Roubidoux, N. R. Dunnick \\ Department of Radiology, University of Michigan Medical Center, 1500 East Medical Center Drive, Ann Arbor, MI 48109-0030, USA
}

With advances in diagnostic imaging, small renal masses are being detected earlier and with increased frequency. The differential diagnosis of a small renal mass is extensive and includes a cyst, infarction, focal infection, angiomyolipoma, lymphoma, metastatic neoplasm, oncocytoma, renal cell carcinoma, and pseudotumor [1]. Differentiation is based on the clinical history and specific imaging findings.

There are three renal cystic diseases that are strongly associated with solid renal masses. Knowledge of these diseases and the renal neoplasms with which they are associated aids the proper diagnosis and treatment of these patients. In this article, we review acquired cystic kidney disease (ACKD), von Hippel-Lindau disease (VHL), and tuberous sclerosis (TS) and suggest the appropriate diagnostic evaluation when a solid renal mass is discovered.

\section{Acquired cystic kidney disease}

ACKD is characterized by the formation of multiple renal cysts in patients with end-stage renal disease who have no history of hereditary cystic disease [2]. Although the term ACKD was initially applied to the development of multiple cysts in patients on long-term hemodialysis [3-5], ACKD also occurs in patients on peritoneal dialysis and in patients prior to the start of dialysis [6].

There are three commonly used nomenclatures for this disease: acquired cystic disease of the kidneys, acquired cystic kidney disease, and acquired renal cystic disease. The occurence of ACKD in patients with renal failure was first noted by Simon in 1847 [7]. However, it was not until 1977, that Dunnill et al. described "acquired cystic disease of the kidneys" in hemodialysis patients [4]. They reported an autopsy series in which $46.6 \%$ of patients on long-term hemodialysis developed renal cysts. They also noted that these patients had two significant complications, perinephric hemorrhage and renal tumor formation. ACKD is now a well-recognized clinical entity with the potential complications of renal cyst hemorrhage, cyst infection, matrix stone formation, renal cell carcinoma, and retroperitoneal bleeding [810].

End-stage renal disease (ESRD) is essential to the development of ACKD. Prior to the initiation of dialysis, as many as $8-22 \%$ of uremic patients develop ACKD [11-14]. Cyst formation is linked to the duration of dialysis. After 1-3 years of dialysis, the prevalence of ACKD is $10-20 \%$, rising to $40-60 \%$ after $3-5$ years, and to more than $90 \%$ after 5-10 years of dialysis $[8,15]$. Before the advent of hemodialysis, these patients had a rapidly fatal disease. It is likely that cyst formation is promoted by dialysis because their improved survival gives these patients a greater opportunity to develop cysts [16-18].

Neither the type of dialysis nor the nature of the underlying renal disease is a factor in the development of renal cyst formation. ACKD affects patients on hemodialysis and peritoneal dialysis equally $[8,11,16$, 19]. Any type of renal disease leading to ESRD may be associated with ACKD [9]. It has been reported that diabetic nephropathy is associated with a lower incidence of ACKD [6, 11], but this may be due to the shorter survival of these patients on dialysis. However, analgesic nephropathy and arteriosclerotic nephrosclerosis tend to be more commonly associated with ACKD $[6,11]$. Men and women have a similar prevalence of ACKD, but the severity of cystic transformation is greater in men than in women $[6,8,9,11]$. Patient age is not a factor, but there may be racial differences because African-Americans are more prone to developing cysts than European-Americans $[6,8,9]$.

The natural history of ACKD after renal transplantation is unclear, and there are conflicting reports on the effect of renal transplantation. Recent data suggest that ACKD often persists after renal transplantation and that 


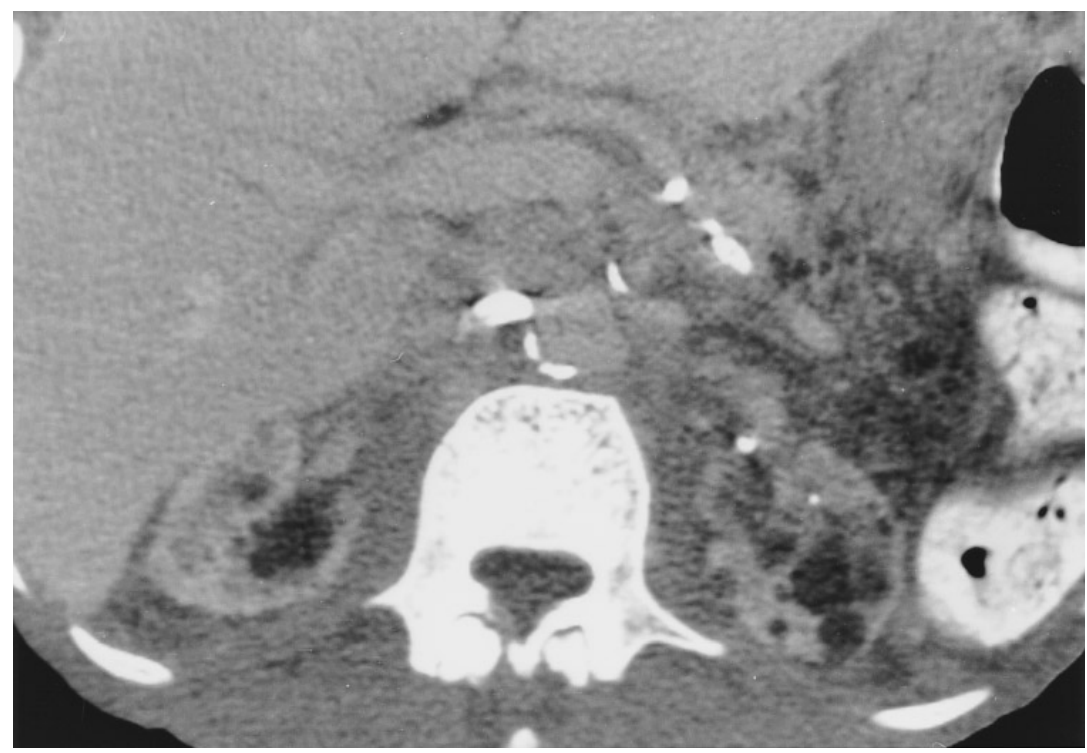

Fig. 1. Acquired cystic kidney disease. Contrast-enhanced CT shows multiple bilateral renal cysts in a patient with ESRD. its persistence is unrelated to the duration of the functioning renal graft $[9,15,20]$. Therefore, renal transplant recipients are still at risk for ACKD and its complications, and this risk increases if the renal transplant function is impaired [8, 9, 15, 21].

\section{Renal pathology}

ACKD is characterized by multiple bilateral small cysts involving both the renal cortex and medulla. On gross examination, the acquired cysts are small, with more than $90 \%$ having a diameter less than $0.6 \mathrm{~cm}$, although some may reach a diameter of $2.5-3.0 \mathrm{~cm}[6,9,16]$. Affected kidneys are usually small, reflecting ESRD, but with cyst formation, the kidneys enlarge $[9,16]$. On histological examination, cysts are typically lined with a single layer of cuboidal or columnar epithelium, although some are lined with multilayered epithelium with papillary projections $[9,16]$.

Renal neoplasms occur with an increased frequency in patients with ACKD [4, 8]. Evidence suggests that renal cyst and renal tumor formation are interrelated. There appears to be a continuum from single-layered epithelial cysts, to multilayered epithelial cysts, to renal cell carcinomas $[9,18]$. Tumors can be multiple and bilateral and often arise from cyst walls. However, renal neoplasms can also occur in areas uninvolved by cysts [16].

Factors causing renal cyst and tumor formation in ESRD are unknown. It is postulated that, with progressive destruction of functioning renal tissue, cystogenic substances accumulate and cause hypertrophy and hyperplasia of epithelial cells $[9,16]$. Focal proliferation of tubular epithelium, oxalate crystal deposition, and surrounding interstitial fibrosis may then result in cystic dilatation of renal tubules $[9,16]$.

\section{Imaging features}

To establish the diagnosis of ACKD, polycystic kidney disease must be excluded, and at least 3-5 renal cysts must be visible on an imaging modality, usually ultrasound, computed tomography (CT), or magnetic resonance $(\mathrm{MR})[6,8,9,11,19,22]$. Depending on the duration of the disease, the radiographic features of ACKD vary widely. Often, no cystic changes are radiographically apparent during the first 3 years of dialysis [19]. Pathologic examination has shown that microscopic cysts are often present during the first few years of dialysis but are too small to be detected radiographically. With time, the cysts grow, enlarging the kidneys and distorting the renal architecture. Ultimately, with progression, these kidneys may resemble those of patients with autosomal dominant polycystic kidney disease [19].

The findings of ACKD on an abdominal radiograph are nonspecific. The kidneys are usually small, the result of chronic renal disease, and cyst wall calcifications can sometimes be detected. Excretory urography is not utilized because these patients have minimal or no renal function.

Ultrasound is useful in the diagnosis and evaluation of ACKD. The affected kidneys have multiple cysts and are usually small with increased echogenicity $[16,19]$. The cysts may cause renal enlargement, and with time the sonographic appearance may resemble autosomal dominant polycystic kidney disease [17, 19]. Ultrasound can also be utilized to help further characterize an indeterminate renal mass discovered on CT. 

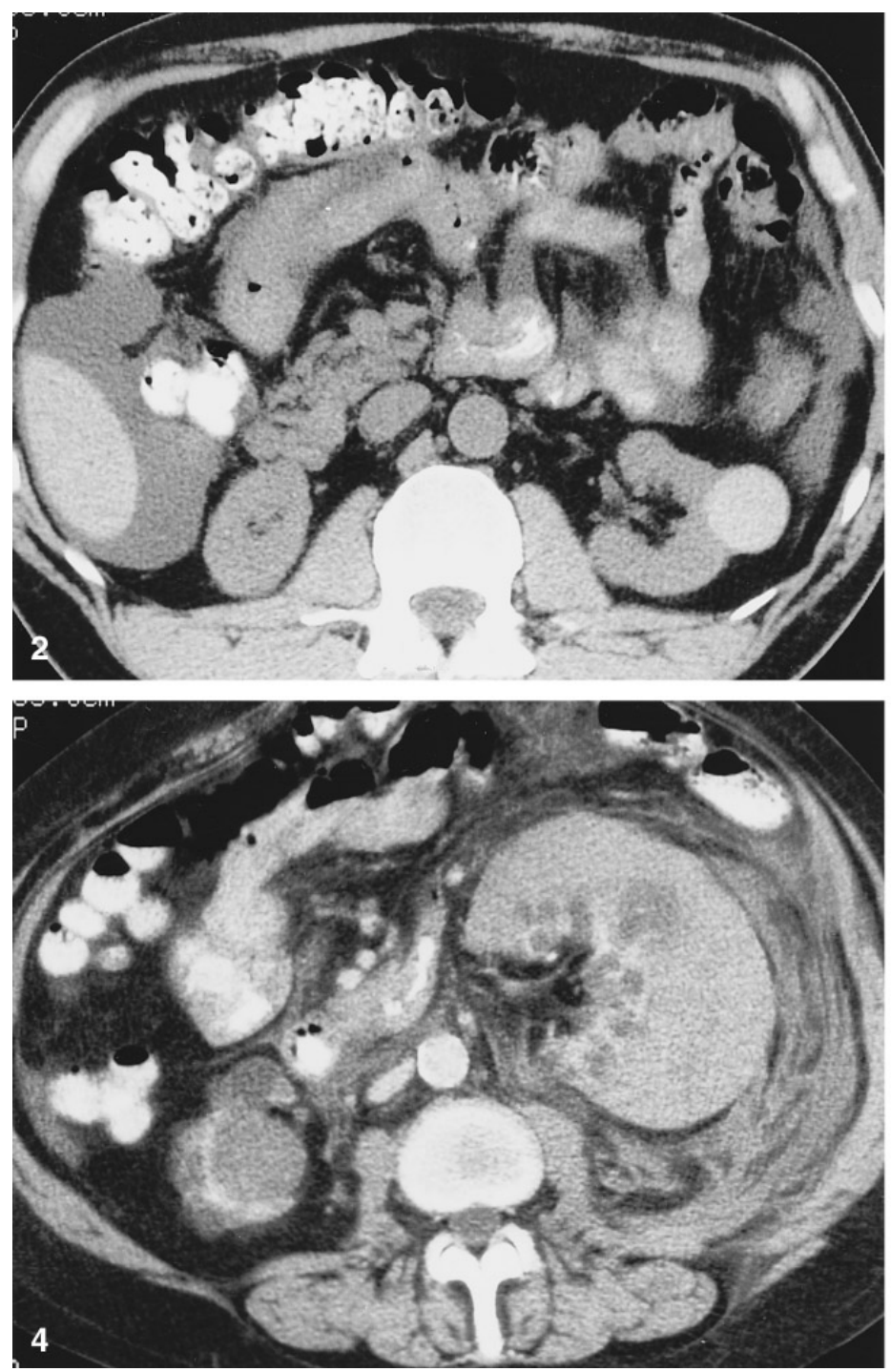

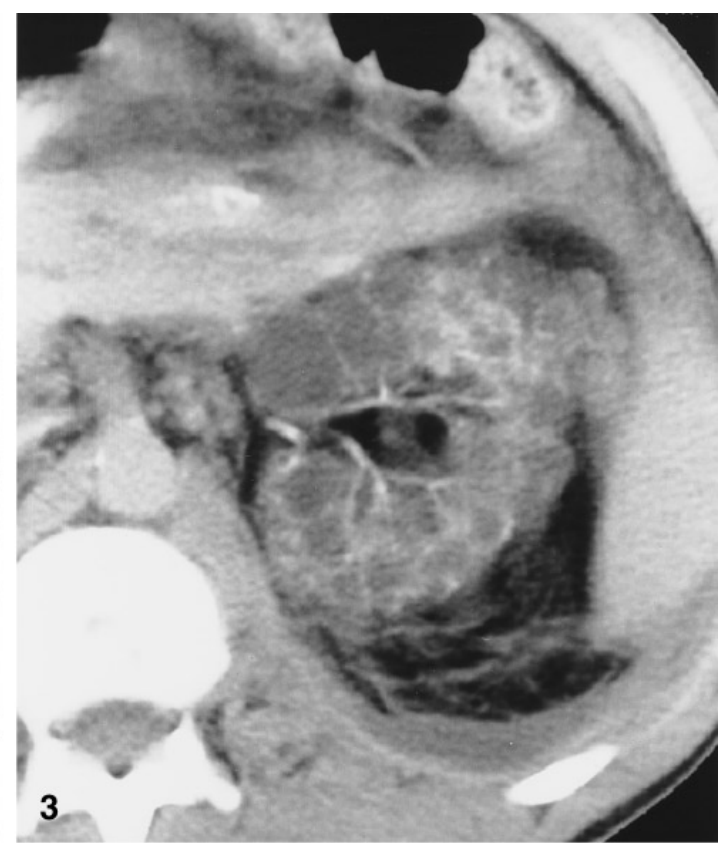

Fig. 2. Acquired cystic kidney disease. Unenhanced CT reveals a high-attenuation, left renal mass; attenuation remained unchanged after intravenous contrast administration. Findings are consistent with a hemorrhagic cyst. Also note intraperitoneal fluid from the patient's peritoneal dialysis.

Fig. 3. Acquired cystic kidney disease. Contrast-enhanced CT shows innumerable renal cysts with an associated left retroperitoneal bleed.

Fig. 4. Acquired cystic kidney disease. Contrast-enhanced CT demonstrates multiple bilateral renal cysts and dystropic calcifications. A large subcapsular hematoma is seen around the left kidney with the fluid in the perinephric space, indicating a perinephric component.
CT is the most sensitive method for detecting ACKD. On CT, the kidneys are studded with multiple small cysts (Fig. 1), and dystrophic cyst wall calcification is common [16]. Iodinated intravascular contrast medium may be used if the patient is on dialysis. However, the referring physician should be alerted because the patient's dialysis schedule may need to be altered to remove the salt load from the administration of intravascular contrast media.

On MR imaging, renal cysts present as multiple, well-defined, nonenhancing, homogeneous masses with low signal intensity on T1-weighted images [23]. MR imaging is not necessary to diagnose ACKD, but it can be helpful in selected patients. MR imaging can be used in patients in whom iodinated intravascular contrast medium is contraindicated; and it can also help further characterize an indeterminate renal mass discovered on CT.

\section{Complications}

The majority of patients with ACKD are asymptomatic [8-10]. The most common presenting complaint is flank pain due to spontaneous hemorrhage. As many as $50 \%$ of patients with ACKD may develop hemorrhagic cysts (Fig. 2) [9]. Hemorrhage into cysts probably occurs because unsupported sclerotic vessels in the cyst walls rupture. Bleeding may also be promoted by systemic heparinization during dialysis and platelet dysfunction due to uremia [9-12]. Bleeding may be confined to the cysts but can also extend into the renal collecting system or the retroperitoneum (Fig. 3). Extension of hemorrhage into the renal collecting system can cause painful gross hematuria. Extension into the subcapsular or perinephric spaces (Fig. 4) can cause flank pain and may be extensive enough to cause a fall in hematocrit. 


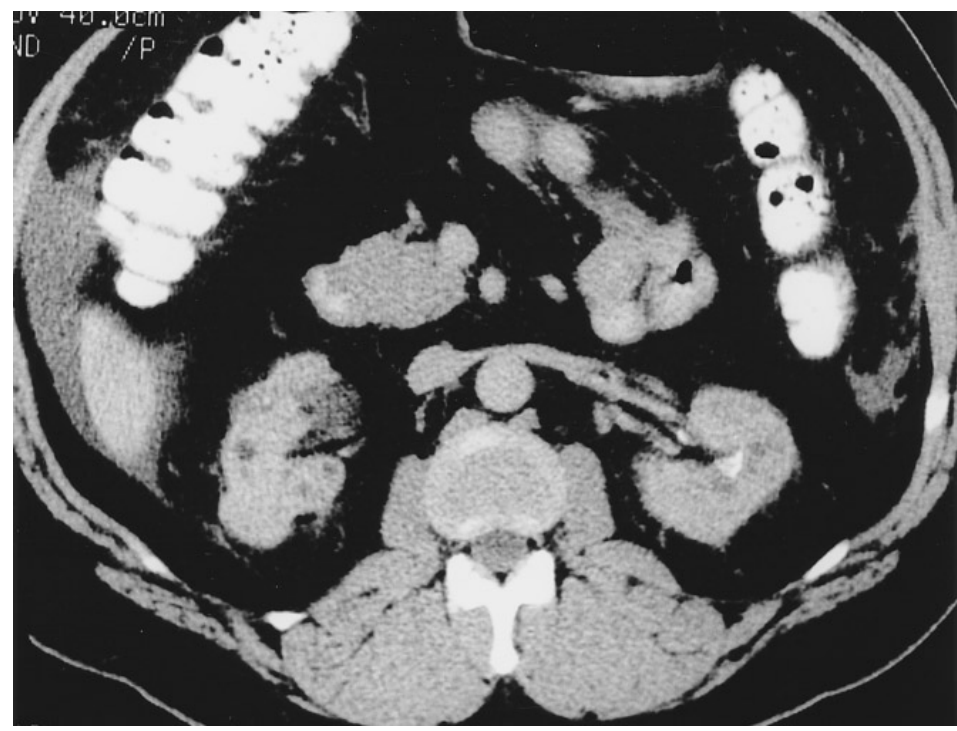

Fig. 5. Acquired cystic kidney disease. Contrast-enhanced CT reveals a solid mass in the right posterior kidney that is a pathologically proven renal cell carcinoma.
The other major complication in ACKD is renal tumor formation (Fig. 5). In patients with ESRD, the incidence of renal cell carcinoma is increased over that of the general population $[8,18,24]$. This risk appears to rise even more in the presence of ACKD [8, 22]. In fact, more than $80 \%$ of patients with ESRD who develop renal cell carcinoma have ACKD [8]. In dialysis patients, renal cell carcinoma presents at an earlier age than in the general population, and it occurs seven times more commonly in men than in women [8,9]. Often these neoplasms are asymptomatic, but sometimes patients present with gross hematuria, flank pain, fever, erythrocytosis, or hypercalcemia $[9,25]$.

It is generally accepted that the incidence of renal cell carcinoma increases in ACKD; however, the degree of increase is controversial. Some investigations have reported an increase in the incidence of renal cancer of greater than 100-fold in dialysis patients over that of the general population $[8,11]$. However, such data do not correlate well with clinical experience. Recent reports have suggested that the annual incidence of invasive or metastatic renal cell carcinoma, that is, "clinically significant' renal cell carcinoma, among dialysis patients is three to six times greater than that of the general population $[2,9,25]$. These data are consistent with a population-based study conducted by the Michigan Kidney Registry in which there was a fivefold increase in the incidence of invasive renal cell carcinoma in dialysis patients as compared with the general population from the same geographic area $[9,25]$.

\section{Diagnostic evaluation}

Some investigators have recommended periodic screening of asymptomatic dialysis patients because of the risk of developing renal cell carcinoma $[5,13,14,18,26-$ 28]. Most have recommended screening beginning after completion of the third year of dialysis because the frequency of ACKD visible on imaging studies significantly increases at this time $[8,9,19]$. In patients undergoing long-term dialysis, the value of routine screening has been questioned because there is insufficient data to assess the effects of screening on patient outcome [9, 15, 25, 29].

In the United States, the overall mortality in dialyzed ESRD patients is high. Patients aged 30-49 years have a life expectancy of 5-10 years, whereas patients 50 years and older have a life expectancy of only $1.5-$ 6 years [9]. These patients die from numerous causes including cardiac disease, cerebrovascular accidents, infection, and withdrawal from dialysis. Malignancy is not a frequently reported cause of death [2]. Therefore, early detection and treatment seems unlikely to significantly improve survival of dialysis patients.

Using a quantitative method of decision analysis, Sarasin et al. assessed the value of routine screening of dialysis patients [30]. Their analysis concluded that routine screening of the entire ESRD population for ACKD and renal malignancy is not justifiable. The strategy of performing either CT or ultrasound examinations every 3 years on all dialysis patients and annually on patients found to have cysts was compared with a strategy of seeking cysts and cancer only if clinically suspected. For patients with a life expectancy of 25 years, screening with either CT or ultrasound examinations may decrease cancer death by half and improve the life expectancy by as much as a 1.6 years [30]. However, age and comorbid disease substantially limit life expectancy for the majority of patients beginning renal replacement therapy. For such patients, an ACKD screening program prolongs average life expectancy only by days [30]. In 
fact, under certain circumstances, an ACKD screening program may even shorten survival of older dialysis patients who are at increased surgical risk $[2,9,30]$. That is, in the presence of $\mathrm{ACKD}$, if the false-positive rate of CT as a test for cancer were $10 \%$, screening would shorten survival for patients aged 64 and older. In the United States, the median age of patients beginning dialysis is 62 years $[9,30]$. Thus, screening could actually shorten the average survival of almost half of ESRD patients in the United States.

Although universal screening of all ESRD patients is not justified, a screening program for selected patients may be beneficial $[9,30]$. Primary considerations for the selection of such patients would include age, general medical condition, life expectancy, and known risk factors, i.e., prolonged dialysis, presence of ACKD, and male gender.

Because there is an increased incidence of renal cell carcinoma in patients with ESRD, the native kidneys of potential transplant candidates should be imaged for renal tumors prior to surgery [2, 21]. However, even though renal transplant recipients remain at risk for ACKD and its complications, the number of case reports of renal cancer developing in the native kidneys of these patients is too small to justify annual screening [2]. Because transplant recipients often undergo ultrasound evaluation of the transplant kidney, some have advocated routinely scanning the native kidneys during this examination [8].

\section{Treatment}

Without complications, ACKD requires no treatment. Patients with symptomatic hemorrhagic cysts, i.e., flank pain and hematuria, can be treated with analgesics. Large hemorrhagic cysts should be followed by serial imaging to document resolution. Because bleeding often subsides spontaneously, retroperitoneal hemorrhage should be managed conservatively with bed rest, hydration, transfusion, correction of coagulation indexes, and analgesics. Patients conservatively treated for perinephric hemorrhage should also undergo serial CT scans to exclude an underlying renal tumor as the cause of the hemorrhage. If a patient continues to bleed despite conservative measures, selective angiography may localize sites of active hemorrhage; selective embolization may stop the bleeding and obviate the need for nephrectomy.

Renal neoplasms can pose significant management problems. If a large renal cell carcinoma is discovered, a nephrectomy should be performed. If a tumor is less than $3 \mathrm{~cm}$ in diameter, management decisions are based on the patient's age, general medical condition, and life expectancy. The potential for these small neoplasms to metastasize is low $[4,12]$. Surgery should be avoided if the patient is a poor operative risk. If CT shows a change in tumor size or appearance, surgical exploration should be performed. In patients who are acceptable operative risks, management of small $(<3 \mathrm{~cm})$ renal neoplasms is controversial. Some have advocated radical nephrectomy in all of these patients, whereas others have recommended monitoring with annual CT scans $[9,11]$.

Because tumors are often multiple and bilateral, there is controversy over whether only the affected kidney should be resected or both kidneys should be removed. In dialysis patients, the risk of developing renal neoplasms in the remaining kidney must be weighed against the increased morbidity and mortality of bilateral nephrectomy. Complications of bilateral nephrectomy include anemia resulting from the loss of endogenous erythropoietin, severe hypotension, and increased dependence of dialysis. Given the risks and benefits, preventive bilateral nephrectomy is not advised.

Renal cell carcinoma does not disqualify a potential transplant candidate, but bilateral nephectomy has been recommended in these patients $[6,9]$. Patients with Robson's stage 1 classification can undergo transplantation immediately, but those with stage 2 need to remain in that stage for 1-2 years prior to renal transplantation [6].

\section{Von Hippel-Lindau disease}

VHL is a rare inherited disorder characterized by a variety of benign and malignant neoplasms throughout the body. VHL has an autosomal dominant mode of inheritance with penetrance approaching $100 \%$ [31, 32]. The most common neoplasms found in patients with VHL are retinal angiomatosis, cerebellar hemangioblastomas, renal cell carcinomas, and pheochromocytomas [33, 34]. Other common neoplasms include other central nervous system hemangioblastomas; angiomas of the liver and kidney; adenomas of the epididymis; pancreatic islet cell tumors; and cysts of the kidney, pancreas, and epididymis [35-38]. Central nervous system hemangioblastomas are the immediate cause of death in more than half of all affected patients, whereas renal carcinomas account for approximately $30 \%$ of deaths $[39,40]$.

Renal cell carcinoma is found in $25-45 \%$ of patients with VHL, is bilateral in $75 \%$ of patients and multifocal in $87 \%[16,32,37,40,41]$. Renal tumors are usually diagnosed in the fourth or fifth decade of life, which is earlier than sporadic cases, which typically arise in the sixth decade [42, 43]. The close association of VHL with renal cell carcinoma led to the localization of the mutant von Hippel-Lindau gene [44]. The gene for VHL has been mapped to chromosome $3 \mathrm{p} 25$, a region often detected in sporadic cases of renal cell carcinoma [45].

Multiple renal cysts occur with increased frequency and are the most common single manifestation of VHL, 


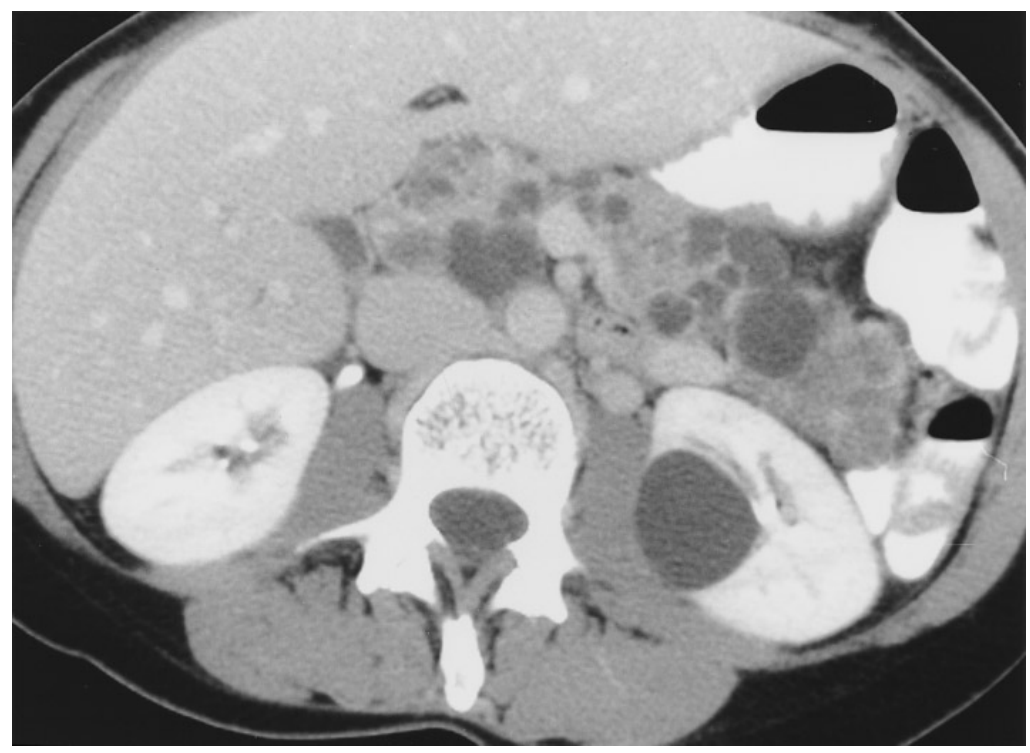

Fig. 6. Von Hippel-Lindau disease. Contrastenhanced CT shows multiple pancreatic and renal cysts in a patient with VHL. occurring in $76 \%$ of patients $[16,37]$. The cysts are typically small and seldom cause renal enlargement. However, uncommonly, the kidneys may enlarge and resemble autosomal dominant polycystic kidney disease [34]. Renal cysts may be the earliest sign of VHL, with renal carcinoma tending to occur late in the disease [16, 34].

\section{Renal pathology}

Occurring mainly in the renal cortex, cysts are typically multiple, bilateral, and small, ranging in size from 0.5 to $3.0 \mathrm{~cm}$ in diameter $[16,46]$. Most cysts are lined by simple cuboidal epithelium and are filled with clear fluid. However, some cystic masses are really renal cell carcinomas that have undergone cystic degeneration. These cystic carcinomas are lined by malignant clear cells similiar to those seen in solid tumors [16].

Renal cell carcinomas are also usually multiple, bilateral, and small, with most carcinomas less than $3 \mathrm{~cm}$ in diameter. Tumors often show irregular margins even though they may have fibrous capsules. Typically, these neoplasms are composed of moderately well-differentiated clear cells; they often show hemorrhage, necrosis, or focal capsular invasion [16].

\section{Imaging features}

Because the kidneys are usually normal in size, abdominal radiographs are often normal. On excretory urography, renal cortical cysts are seen bilaterally. The cysts distort the renal architecture, making it difficult to diagnose small tumors unless they are peripheral or displace the collecting system [32, 47]. Thus, excretory urography is seldom used in the evaluation of patients with VHL.

Ultrasound is highly sensitive for the detection of renal cysts. Because it is readily available, relatively inexpensive, and does not require ionizing radiation, it is often used to detect renal cysts. Ultrasound may also help in the evaluation of an indeterminate lesion seen on CT. In patients with known VHL, ultrasound is of limited use for screening because it is insensitive in detecting small renal tumors.

CT is more sensitive than ultrasound in the detection of renal masses. Furthermore, lesions can be precisely defined, which facilitates monitoring lesion growth. Cysts are characterized by well-defined, thin-walled, nonenhancing, fluid attenuation masses (Fig. 6). Solid renal masses typically have indistinct irregular tumor margins, heterogeneity, and contrast enhancement that is less than that seen in normal renal parenchyma (Figs. $7,8)$.

MR imaging is indispensible for diagnosing and evaluating the multiple neurological neoplasms common in patients with VHL; however, MR of the kidneys is of limited use. Unless they alter the shape of the kidneys, small renal cell carcinomas can go undetected because these lesions tend to have a signal intensity similar to that of renal parenchyma on both $\mathrm{T} 1$ - and $\mathrm{T} 2$ weighted images [23]. Gadolinium administration greatly improves detection because enhancement of renal cell carcinoma differs from that of normally enhancing renal parenchyma [23]. Large tumors are more easily recognizable, tend to be more heterogeneous, and sometimes contain areas of hyperintensity on $\mathrm{T} 1-$ and T2-weighted images [23].

The angiographic findings of renal cell carcinoma in patients with VHL include well-circumscribed, mul- 

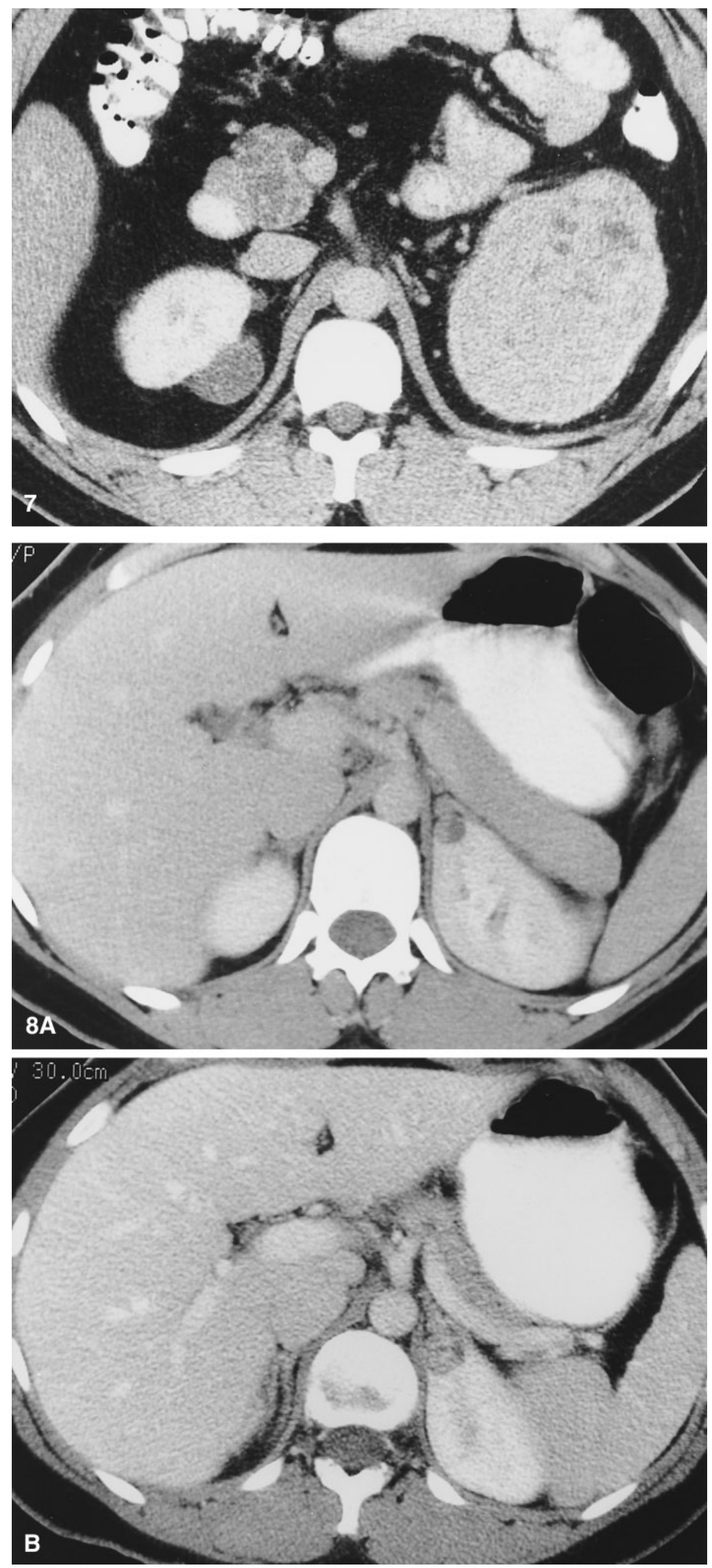

Fig. 7. Von Hippel-Lindau disease. Contrast-enhanced CT demonstrates a huge left renal mass and a smaller solid mass in the right kidney. On pathological examination, both were renal adenocarcinoma. Also note innumerable pancreatic cysts.

Fig. 8. Von Hippel-Lindau disease. A Contrastenhanced CT reveals a small left renal mass. B Images obtained 12 months later show interval growth of this renal cell carcinoma. 
ticentric lesions with neovascularity [32, 40]. Even selective renal angiography does not always detect small renal tumors, nor can it reliably differentiate benign from malignant lesions [40]. Therefore, angiography is seldom used for the detection or exclusion of renal cell carcinoma. Renal angiography is primarily limited to vascular mapping prior to partial nephrectomy or tumor enucleation [40].

\section{Diagnostic evaluation}

To find renal cell carcinomas while they are still small and before they have metastasized, patients with VHL should be screened for asymptomatic renal tumors. Some have suggested MR imaging as a potentially useful screening examination [31]; however, CT is the most frequently used method of screening because it demonstrates more renal lesions and is less expensive than MR [41]. Most have advocated annual screening of all patients with VHL even in the absence of abdominal symptoms [34, 42, 48]. Some have recommended screening every $2-4$ years if the initial CT examination is normal $[16,45]$.

Because VHL has an autosomal dominant mode of inheritance, half of the offspring of an affected patient may be expected to develop the disease. Periodic screening of at-risk family members is essential. There is no consensus as to the age at which screening should commence, the frequency with which it should be performed, or the modality that should be used. Some have advocated beginning screening at preschool age, with repeat scans every $2-3$ years up to age 20 years and then annually into the sixth or seventh decade [38]. Others have recommended initial scanning when patients are 18-20 years old because most disease manifestations become apparent at that age and repeat scans every 1-5 years, depending on the imaging findings and the disease pattern in the family $[16,39]$. The recommended screening modality is $\mathrm{CT}$, but abdominal ultrasound may be used for patients $<16$ years old [35]. If an atrisk family member is diagnosed with VHL, the patient should be followed more closely.

\section{Treatment}

Renal cysts are usually asymptomatic and require no therapy. However, apparent cysts are sometimes renal cell carcinomas that have undergone cystic degeneration. Therefore, a change in any renal mass is an indication for further evaluation and possible surgery. Because the lesions are sometimes smaller than $1 \mathrm{~cm}$, distinction between small renal cysts and carcinomas often can not be made. If CT identifies a small indeterminate mass, annual CT examinations using narrow collimation are recommended [16, 37].
Renal cell carcinoma requires surgical management. However, because renal neoplasms are often bilateral and multicentric in patients with VHL, treatment has been controversial. Surgical alternatives include renalsparing tumor enucleation, partial nephectomy, and bilateral nephrectomy with hemodialysis or transplantation [35, 38, 42, 49, 50]. Standard nephrectomy would eventually lead to many anephric patients. With renalsparing enucleation or partial nephectomy, patients can live without dialysis or transplantation, but there is a $75 \%$ chance of recurrence within the same or opposite kidney [38]. The risks and complications of bilateral nephrectomy must be weighed against the risk of recurrent renal cell carcinoma. A conservative surgical approach has been adopted because renal-conserving procedures preserve renal function and spare patients the morbidity and mortality associated with hemodialysis and transplantation [40-42, 48-51]. In patients in whom bilateral nephrectomies are unavoidable, a delay in renal transplantation for at least 1 year has been advocated $[39,41]$.

\section{Tuberous sclerosis}

TS, formerly known as Bourneville's disease, is a genetically transmitted neurocutaneous disorder characterized by mental retardation, seizures, and cutaneous lesions. TS has an autosomal dominant mode of inheritance, with a high penetrance and variable expressivity [52]. In addition, some cases are sporadic and apparently the result of spontaneous new mutations $[16,53]$. This disorder is characterized by benign congenital tumors (hamartomas) composed of normal tissues but in abnormal quantities, arrangement, maturation, and growth. These hamartomas occur in the brain (cortical tubers), retina, skin, heart (rhabdomyomas), bone, lung, liver, adrenal gland, pancreas, and kidney (angiomyolipomas) [52-54].

The most common of these hamartomas is renal angiomyolipomas, which has an incidence of $70-95 \%$ among patients with TS [16]. Furthermore, the incidence appears to increase with age $[52,55]$. Less commonly known is the renal cyst formation that also occurs in TS. Renal cysts occur less frequent than angiomyolipomas and can occur alone or in conjunction with angiomyolipomas [54]. Rarely, renal cystic disease may be the earliest clinical manifestation of TS in childhood [45, 53-56].

The occurrence of both angiomyolipomas and renal cysts is virtually diagnostic of TS. These lesions can be unilateral and solitary but are typically multiple and bilateral. Angiomyolipomas and renal cysts are usually asymptomatic, but in some patients these lesions cause significant problems. Angiomyolipomas may spontaneously bleed, and renal cysts can increase in number 


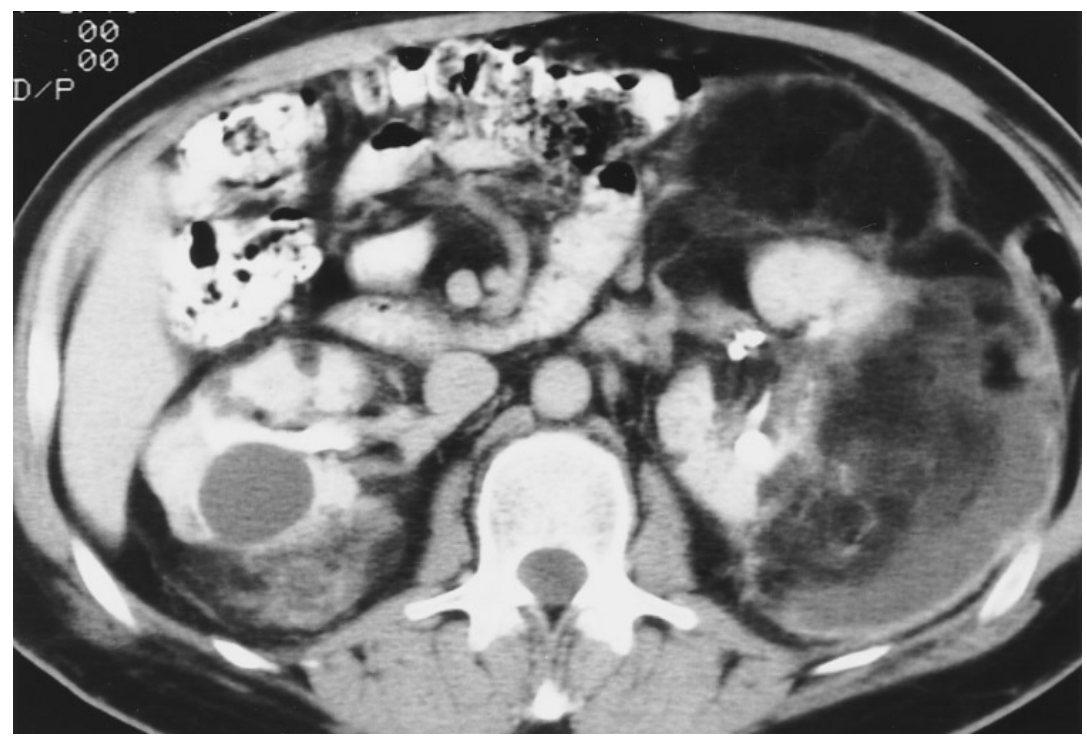

Fig. 9. Tuberous sclerosis. Contrast-enhanced CT demonstrates multiple renal cysts and bilateral fatty masses consistent with angiomyolipomas. and size significant enough to cause renal insufficiency $[53,54,57]$. Five to 15 percent of TS patients develop ESRD [45]. ESRD is more likely when renal cysts are present (with or without angiomyolipomas) than when angiomyolipomas are present alone [57]. Whether or not there is an increased incidence of renal cell carcinoma is controversial $[16,45,53,57]$.

\section{Renal pathology}

Renal cysts vary in size, from microscopic to $3 \mathrm{~cm}$ in diameter. These cysts, which are probably of tubular origin, diffusely involve the kidneys. The cysts usually have an unique epithelial lining composed of large cells with eosinophilic cytoplasm and prominent hyperchromatic nuclei $[16,54,57]$. Because renal cysts may be the first clinical manifestation of TS, these unique histological findings can strongly suggest the diagnosis.

Angiomyolipomas are benign nonencapsulated masses consisting of varying amounts of abnormal blood vessels, smooth muscle, and mature fat. The majority of angiomyolipomas are small, less than $1 \mathrm{~cm}$ in diameter [52]. Hemorrhage and necrosis are common, and calcifications can occur $[45,58]$. Smooth muscle cells may show hyperchromatism, pleomorphism, and occasional mitotic figures; however, these findings are insufficient for the diagnosis of malignancy [54, 57].

\section{Imaging features}

On abdominal radiographs, lucency suggesting fat within an angiomyolipoma can occasionally be detected. The findings on excretory urography and retrograde pyelography depend on the number and size of cysts and angiomyolipomas. Findings range from normal to an appearance that mimics autosomal dominant polycystic kidney disease. On excretory urography, there are typically multiple nonopacifying renal masses, with some distortion of the collecting system.

On sonographic examination, renal cysts are anechoic and multiple. The sonographic appearance of an angiomyolipoma depends on its fat content. If there is abundant fat, angiomyolipomas are hyperechoic; however, if predominately nonfatty, these cysts are indistinguishable from other solid tumors.

CT is the most useful imaging modality for evaluating the kidneys. Renal cysts appear identical to simple cysts, and some may contain mural calcifications (Fig. 9). If the kidneys enlarge, CT findings can be indistinguishable from autosomal dominant polycystic kidney disease. However, unlike autosomal dominant polycystic kidney disease, the renal cysts are usually associated with angiomyolipomas. The key to diagnosing an angiomyolipoma is the demonstration of fat. Because of its sensitivity in detecting small foci of fat, CT is the optimal imaging modality. Fat-containing angiomyolipomas have low (negative) attenuation values. However, if angiomyolipomas are predominately nonfatty or if intramural hemorrhage obscures the fat, they are indistinguishable from other solid tumors.

On MR, renal cysts are seen as multiple, well-defined, nonenhancing, homogeneous masses with low signal intensity on T1-weighted images [23]. Because they contain fat, angiomyolipomas have high signal intensity on both T1- and T2-weighted images. MR imaging is seldom needed in the diagnosis of the renal lesions; however, MR imaging can help further characterize and evaluate an indeterminate mass discovered on CT. 

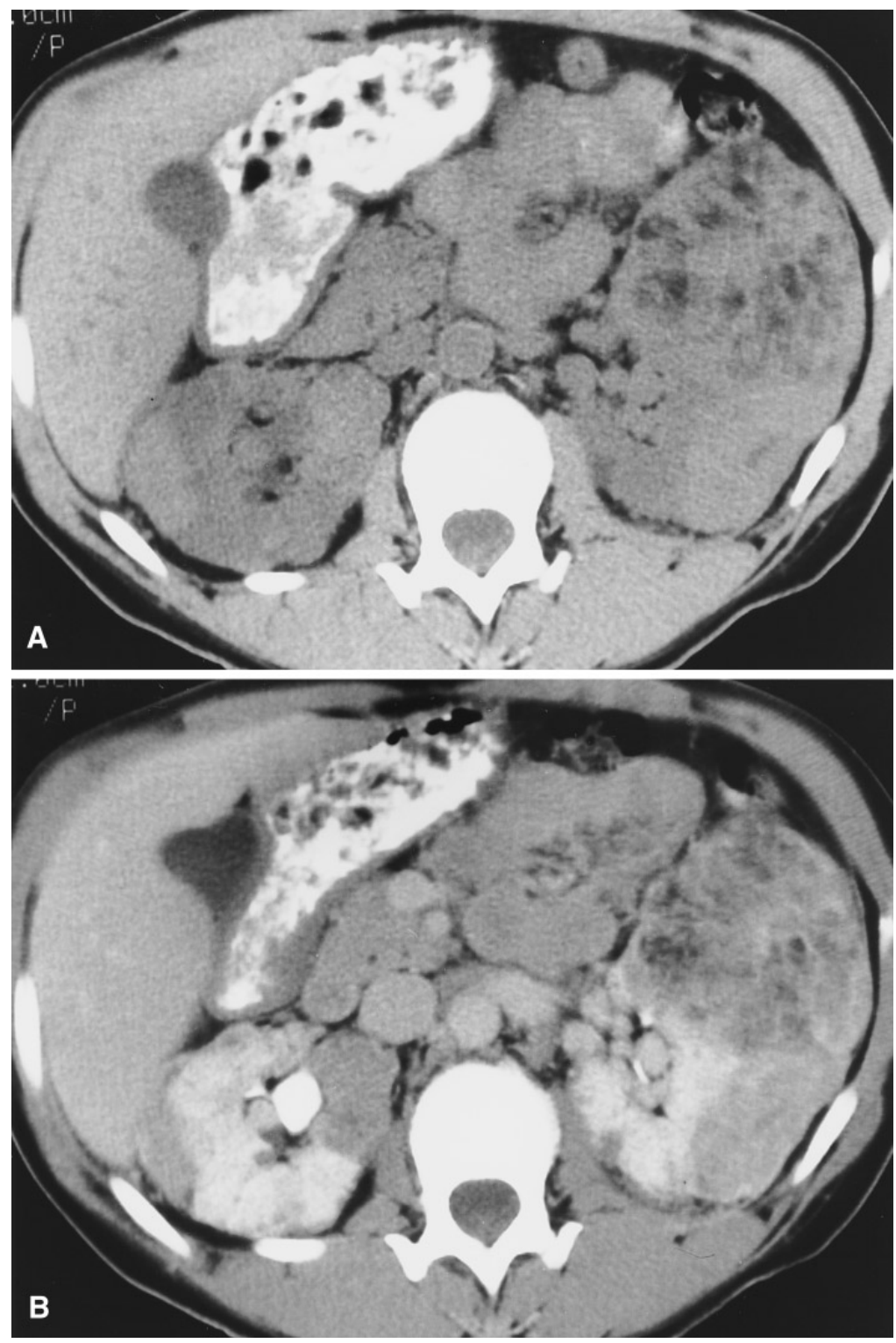

Fig. 10. Tuberous sclerosis. A Unenhanced CT shows angiomyolipomas and areas of increased attenuation. B After intravenous contrast administration, the lack of enhancement of these high attenuation fluid collections indicates subcapsular hematoma. Also note the water attenuation cysts apparent on the enhanced images.
Renal angiography is seldom used for diagnostic purposes but can be helpful in the evaluation of complications. If performed, multiple avascular zones corresponding to renal cysts can be seen. The angiographic appearance of angiomyolipomas is variable depending on the amount of angiomatous tissue. Most angiomyolipomas are recognized by their small aneurysms and whorled nephrogram of neovascularity $[16,59]$.

\section{Diagnostic evaluation}

In patients without other stigmata of TS, angiomyolipomas can provide a clue to the diagnosis of TS because angiomyolipomas do not occur in other renal cystic diseases. Careful examination using CT with narrow col- limation can often show small fat-containing angiomyolipomas. Because angiomyolipomas are not always present, absence of angiomyolipomas does not exclude the diagnosis of TS. Rarely, renal cysts are the only renal manifestation [16].

A variation on the renal cystic disease of TS is a subgroup of patients who have severe cystic disease grossly indistinguishable from autosomal dominant polycystic kidney disease [54-57]. Because of this subgroup, it has been recommended that all children presenting with the clinical or radiological appearance of polycystic kidney disease should receive a renal CT with narrow collimation searching for angiomyolipomas or a renal cyst biopsy to evaluate for hyperplastic eosinophilic epithelial lining [54-56]. These patients should also receive a thorough evaluation to search for other lesions of TS. 


\section{Treatment}

Renal cystic disease of TS is usually asymptomatic, and treatment is rarely necessary. Treatment is only necessary when the cysts become large and cause pain, obstruction, and hemorhage [54]. As renal lesions grow, they compress and replace renal parenchyma and thus diminish renal function. Therefore, in select cases, some have advocated decompression of the cysts to protect adjacent renal parenchyma from atrophy [53, 54]. Some patients with renal cystic disease develop hypertension and renal insufficiency progressing to ESRD. In these patients, antihypertensive medication and dialysis may become necessary.

Most angiomyolipomas are asymptomatic. The main complication of angiomyolipomas is spontaneous hemorrhage (Fig. 10). The risk of hemorrhage increases with the size of the angiomyolipoma [60, 61]. Angiomyolipomas associated with TS grow faster than do sporadic angiomyolipomas [45, 60]. An actively bleeding angiomyolipoma can be treated by selective angiography and embolization of the intrarenal artery supply in the tumor [53, 59]. In life-threatening bleeds that are not amendable to angiography, surgical intervention becomes necessary. Functioning renal parenchyma is already compromised by replacement of the renal parenchyma with cysts and angiomyolipomas; therefore, partial nephectomy or encleation should be attempted to preserve functioning renal parenchyma. Because large $(>4 \mathrm{~cm})$ angiomyolipomas are at increased risk of hemorrhage, they can be carefully observed, prophylactically embolized, or surgically resected. Often, an aggressive approach with selective embolization is advised for larger angiomyolipomas [61]. Cases of renal cell carcinoma associated with TS have been reported. Therefore, renal cancer should be suspected in cases of renal lesions without demonstrable fat [53]. If malignancy is suspected, exploratory surgery is necessary.

\section{References}

1. Bosniak M. The small $(<3.0 \mathrm{~cm})$ renal parenchymal tumor: detection, diagnosis and controversies. Radiology 1991;179:307317

2. Levine E. The query corner: acquired cystic kidney disease. $A b$ dom Imaging 1995;20:569-573

3. Scanlon M, Karasick S. Acquired renal cystic disease and neoplasia: complications of chronic hemodialysis. Radiology 1983; 147:837-838

4. Dunnill MS, Millard PR, Oliver D. Acquired cystic disease of the kidney: a hazard of long-term intermittent maintenance haemodialysis. J Clin Pathol 1977;30:868-877

5. Weissberg D, Miller R. Renal cell carcinoma and acquired cystic disease of the kidneys in a chronically dialyzed patient. J Ultrasound Med 1983;2:191-194

6. Ishikawa I. Uremic acquired renal cystic disease. Nephron 1991;58:257-260
7. Simon J. On Sub-acute inflammation of the kidney. Medico Chir Trans 1847;30:141-164

8. Matson M, Cohen E. Acquired cystic kidney disease: occurrence, prevalence, and renal cancers. Medicine 1990;69:217-225

9. Levine E. Acquired cystic kidney disease. Radiol Clin North Am 1996;34:947-964

10. Soffer O, Miller L, Lichtman J. CT findings in complications of acquired renal cystic disease. $J$ Comput Assist Tomogr 1987;11:905-908

11. Chandhoke P, Torrence R, Clayman R, et al. Acquired cystic disease of the kidney: a management dilemma. $J$ Urol 1992;147:969-974

12. Levine E, Grantham J, Slusher S, et al. CT of acquired cystic kidney disease and renal tumors in long-term dialysis patients. AJR 1984;142:125-131

13. Henson JHL, Al-Hilli A, Penry JB, et al. The development of acquired renal cystic disease and neoplasia in a chronic haemodialysis patient. Br J Radiol 1985;58:1215-1217

14. Kutcher R, Amodio J, Rosenblatt R. Uremic renal cystic disease: value of sonographic screening. Radiology 1983;147:833-835

15. Heinz-Peer G, Schoder M, Rand T, et al. Prevalence of acquired cystic kidney disease and tumors in native kidneys of renal transplant recipients: a prospective US study. Radiology 1995;195:667-671

16. Levine E, Hartman D, Smirniotopoulos J. Renal cystic disease associated with renal neoplasms. In: Pollack H, ed. Clinical urography. Philadelphia: WB Saunders, 1990:1126-1150

17. Andersen B, Curry N, Gobien R. Sonography of evolving renal cystic transformation associated with hemodialysis. AJR 1983;141:1003-1004

18. Bretan P, Busch M, Hricak H, et al. Chronic renal failure: a significant risk factor in the development of acquired renal cysts and renal cell carcinoma. Cancer 1986;57:1871-1879

19. Jabour B, Ralls P, Tang WW, et al. Acquired cystic disease of the kidneys computed tomography and ultrasonography appraisal in patients on peritoneal and hemodialysis. Invest Radiol 1987;22:728-732

20. Lien Y, Hunt KR, Siskind M, et al. Association of cyclosporin A with acquired cystic kidney disease of the native kidney in renal transplant recipients. Kidney Int 1993;44:613-616

21. Levine L, Gburek B. Acquired cystic disease and renal adenocarcinoma following renal transplantation. J Urol 1994;151:129_ 132

22. Takase K, Takahashi S, Tazawa S, et al. Renal cell carcinoma associated with chronic renal failure: evaluation with sonographic angiography. Radiology 1994;192:787-792

23. Rominger M, Kenney P, Morgan D, et al. Gadolinium-enhanced MR imaging of renal masses. Radiographics 1992;12:1097-1116

24. Siegel S, Sandler M, Alpern M, et al. CT of renal cell carcinoma in patients on chronic hemodialysis. AJR 1988;150:583-585

25. Levine E, Renal cell carcinoma in uremic acquired renal cystic disease: incidence, detection, and management. Urol Radiol 1992;13:203-210

26. Heinz-Peer G, Helbich T, Nottling B, et al. Renal cell carcinoma in an allograft kidney transplant. Transplantation 1994;57:475478

27. Munechika H, Kitanosono T, Sato S, et al. Bilateral multiple renal adenocarcinomas in a patient with acquired renal cystic disease: correlation of imaging with pathological study. Urol Radiol 1992;13:146-152

28. Taylor AJ, Cohen EP, Erickson SJ, et al. Renal imaging in longterm dialysis patients: a comparison of CT and sonography. AJR 1989;153:765-767

29. Mindell H. Imaging studies for screening native kidneys in longterm dialysis patients. AJR 1989;153:768-769

30. Sarasin FP, Wong JB, Levey AS, et al. Screening for acquired cystic kidney disease: a decision analytic perspective. Kidney Int 1995;48:207-219 
31. Sato Y, Waziri M, Smith W, et al. Hippel-Lindau disease: MR imaging. Radiology 1988;166:241-246

32. Fill W, Lamiell J, Polk N. The radiographic manifestations of von Hippel-Lindau disease. Radiology 1979;133:289-295

33. Goodman MD, Goodman B, Lubin M, et al. Cytogenetic characterization of renal cell carcinoma in von Hippel-Lindau syndrome. Cancer 1990;65:1150-1154

34. Lee D. Renal cystic diseases. In: Taveras J, Ferrucci J, eds. Radiology diagnosis - imaging - intervention, vol. 4. Philadelphia: JB Lippincott, 1991:1-12

35. Choyke P, Filling-Katz M, Shawker T, et al. Von Hippel-Lindau disease: radiologic screening for visceral manifestations. Radiology 1990;174:815-820

36. Hull M, Warfel K, Muller J, et al. Familial islet cell tumors in von Hippel-Lindau's disease. Cancer 1979;44:1523-1526

37. Levine E, Collins D, Horton W, et al. CT screening of the abdomen in von Hippel-Lindau disease. AJR 1982;139:505-510

38. Malek R, Omess P, Benson R, et al. Renal cell carcinoma in von Hippel-Lindau syndrome. Am J Med 1987;82:236-238

39. Lamiell J, Salazar F, Hsia E, Von Hippel-Lindau disease affecting 43 members of a single kindred. Medicine 1989;68:1-29

40. Miller D, Choyke P, Walther M, et al. Von Hippel-Lindau disease: inadequacy of angiography for identification of renal cancers. Radiology 1991;179:833-836

41. Lund G, Fallon B, Curtis M, et al. Conservative surgical therapy of localized renal cell carcinoma in von Hippel-Lindau disease. Cancer 1994;74:2541-2545

42. Levine E, Weigel J, Collins D. Diagnosis and management of asymptomatic renal cell carcinomas in von Hippel-Lindau syndrome. Urology 1983;21:146-150

43. Sharif HS, Furneaux C, Srivatsa SR. Unusual urographic findings in a case of von Hippel-Lindau disease. Br J Radiol 1983;56:132136

44. Caviness VS. Neurocutaneous syndromes and other developmental disorders of the central nervous system. In: Wilson JD, Braunwald $\mathrm{E}$, Isselbacher KJ, et al., eds. Harrison's principles of internal medicine. New York: McGraw-Hill, 1991:2056

45. Choyke P. Inherited cystic disease of the kidney. Radiol Clin North Am 1996;34:925-946

46. Lee K, Wulfsberg E, Kepes J. Some important radiological aspects of the kidney in Hippel-Lindau Syndrome: the value of pro- spective study in an affected family. Radiology 1977;122:649653

47. Levine E, Lee $\mathrm{K}$, Weigel $\mathrm{J}$, et al. Computed tomography in the diagnosis of renal carcinoma complicating Hippel-Lindau syndrome. Radiology 1979;130:703-706

48. Frydenberg M, Malek RS, Zincke H. Conservative renal surgery for renal cell carcinoma in von Hippel-Lindau's disease. J Urol 1993;149:461-464

49. Loughlin K, Gittes R. Urological management of patients with von Hippel-Lindau's disease. J Urol 1986;136:789-791

50. Nelson JB, Oyasu R, Dalton DP. The clinical and pathological manifestations of renal tumors in von Hippel-Lindau disease. $J$ Urol 1994;152:2221-2226

51. Shinohara N, Nonomura K, Harabayashi T, et al. Nephron sparing surgery for renal cell carcinoma in von Hippel-Lindau disease. $J$ Urol 1995;154:2016-2019

52. Bell D, King B, Hattery R, et al. Imaging characteristics of tuberous sclerosis. AJR 1991;156:1081-1086

53. Torres VE, King BF, Holley KE, et al. The kidney in the tuberous sclerosis complex. Adv Nephrol 1994;23:43-70

54. Stillwell T, Gomez M, Kelalis P. Renal lesions in tuberous sclerosis. J Urol 1987;138:477-481

55. Mitnick J, Bosniak M, Hilton S, et al. Cystic renal disease in tuberous sclerosis. Radiology 1983;147:85-87

56. Campos A, Figueroa ET, Gunasekaran S, et al. Early presentation of tuberous sclerosis as bilateral renal cysts. J Urol 1993;149:1077-1079

57. Bernstein J, Robbins TO, Kissane JM. The renal lesions of tuberous sclerosis. Sem Diagn Pathol 1986;3:97-105

58. Sherman J, Hartman D, Friedman A, et al. Angiomyolipoma: computed tomographic-pathologic correlation of 17 Cases. AJR 1981;137:1221-1226

59. Bosniak M. Angiomyolipoma (hamartoma) of the kidney: a preoperative diagnosis is possible in virtually every case. Urol Radiol 1981;3:135-142

60. Lemaitre L, Robert Y, Dubrulle F, et al. Renal angiomyolipoma: growth followed up with CT and/or US. Radiology 1995; 197:598-602

61. Van Ball JG, Smits NJ, Keeman JN, et al. The evolution of renal angiomyolipomas in patients with tuberous sclerosis. J Urol $1994 ; 152: 35-38$ 